\title{
Companion robots for older adults: Rodgers' evolutionary concept analysis approach
}

\author{
Jeongeun $\mathrm{Kim}^{1}$. Sukwha $\mathrm{Kim}^{2}$. Seongheui $\mathrm{Kim}^{3}$ - Euehun Lee ${ }^{4}$ Yoonjeong $\mathrm{Heo}^{5}$. Cheol-Yong Hwang ${ }^{6}$. \\ Yun-Young Choi ${ }^{7} \cdot$ Hyoun-Joong Kong ${ }^{8} \cdot$ Hyeongju Ryu ${ }^{9} \cdot$ Hyeongsuk Lee ${ }^{10}{ }_{\mathbb{C}}$
}

Received: 16 February 2021 / Accepted: 15 October 2021 / Published online: 16 November 2021

(c) The Author(s), under exclusive licence to Springer-Verlag GmbH Germany, part of Springer Nature 2021

\begin{abstract}
This study aims to analyze the concept of companion robots for older adults from the perspective of nursing. This study employed a concept analysis. The literature from July 2011 to June 2021 was sought from databases using specific keywords. Any quantitative or qualitative study published in English or Korean focusing on companion robots for older adults was included in the study. Rodgers' evolutionary concept analysis was used to clarify the antecedents, attributes, and consequences. Seventy-five eligible articles were studied. The findings were categorized into antecedents, attributes, and consequences. Companion robot antecedents were classified into individual factors, attitude toward robots, and caregiver and social factors. The defining attributes included human-robot interaction, function, features, structure, cost, and management of the robot being a companion. Consequences were categorized into user, caregiver, and health related. Companion robots are designed to enhance well-being, quality of life, and independence by providing service and companionship and assisting daily life. This mainly includes cognitive and social support, mobility support, relaxation, health monitoring, and self-care support through human-robot interaction. The attributes, antecedents, and consequences of companion robots identified in this study can inform future decision making and interventions by caregivers for aging in place.
\end{abstract}

Keywords Companion robot $\cdot$ Older adults $\cdot$ Concept analysis $\cdot$ Nursing care $\cdot$ Aging in place

\section{Introduction}

Several countries worldwide are experiencing a rapidly aging population. However, the number of healthcare professionals to care for older adults is low [1]. Furthermore, social isolation - that is, loneliness because of limited social connections-may put older adults' psychological well-being at risk [2]. The COVID-19 crisis worsened this situation, increasing interest in robots that could help create, maintain, and strengthen social relationships [3]. In this context, various technology-driven interventions are being considered alternatives [4]. Information and communication technologies (ICT), assistive technologies (AT), and human-computer interaction (HCI) technologies are interdisciplinary topics being actively pursued in research and clinical practice. Among these, robotics and artificial intelligence (AI) are

\section{Hyeongsuk Lee}

leehyeongsuk@gachon.ac.kr

Extended author information available on the last page of the article expected to substitute mindless mimicry of human behavior for the real presence of conscious caring offered by humans.

Companion robots assist older adults, enabling them to remain autonomous in their residences for as long as possible [5]. Companion robots fall under the broader umbrella of socially assistive companion robots, whose use in the care of older adults has been widely reviewed, but mostly from a quantitative perspective [6, 7] and often across a broad base of care settings not specifically residential care. The literature focusing on robots for older adults has dealt with the concept of "robot" in a context wider than mere companion robots. For instance, the authors of [2] presented the definition of robots for older adults by classifying them into companion, telepresence, rehabilitation, health monitoring, and entertainment robots. Among them, companion robots are expected to be used in interventions for the social isolation of older adults and as a support system for aging in place.

However, considering that gerontechnology involves multiple scientific disciplines, such as geriatric medicine, psychology, social sciences, computer sciences, engineering and 
design, informatics, and economics, it is difficult to establish general, agreed methodological standards for evaluating technology in clinical practice [8]. Proper understanding and use of concepts are important for psychological and physical self-management by incorporating robots into nursing. Therefore, there is a need to reach a consensus about the definition of these technologies.

Furthermore, a paradigm shift has led to a greater variety of multidisciplinary approaches and programming methods in relatively simpler interventions, such as helping older people with their work, which are used in complex contexts for multiple purposes. Therefore, a new concept analysis is required considering the different situations in which the concept of a companion robot for older adults is used and the perceptions in various fields.

\section{Methods}

\subsection{Concept analysis method}

This study uses Rodgers' evolutionary concept analysis [9]. Although there are many ways to analyze concepts, Rodgers' approach is best suited for analyzing companion robots for older people. Over time, the purpose and context of using robots will differ, and new concepts will develop. Rodgers' model is based on Wilson's conceptual analysis method [10] and reflects the changing and dynamic nature of concepts. According to Rodgers [9], "significance" is a central aspect of the concepts chosen for analysis. "Significance" posits that a concept should serve a purposeful human goal in an actual case or praxis, contribute to solving problems, and provide an adequate characteristic of the phenomenon. Several concepts can express the same idea, which can initially confuse and affect the choice of concept analysis. When a concept is defined to a greater degree, it becomes possible to describe the phenomenon and its characteristic manner regarding the distinctive character of a discipline. Rodgers' model discerns interdisciplinary divergences regarding the concept and incorporates those presented in various fields [11]. This method is suitable for analyzing companion robots for older people in need of assistance across domains, such as nursing, medicine, and engineering.

Rodgers' evolutionary method identifies and categorizes data presented in each discipline related to the concept by emphasizing data collection. The process is carried out in six steps: (1) specifying a concept and its alternate terminologies; (2) determination and selection of the appropriate scope for data collection; (3) collection of data related to concept attributes and conceptual basis, including sociocultural, interdisciplinary, and temporal variables; (4) analysis based on concept attributes; (5) provision of examples that fit the concept, if required; and (6) determination of hypotheses and applications for further evolution of the concept [9].

\subsection{Sources of data}

Fields of study related to companion robots for older adults include nursing, medicine, and robotics. Databases, such as CINAHL, MEDLINE, PubMed, and EMBASE, were searched for related articles using "AND" and "OR" operators in titles and abstractions. Search keywords were "aged," "elder*," "older adult*," "older people," "robot*," "friend," "companion," "service," and "assist*." We used "Not" operation, "surgery," or "rehabilita*" as keywords to exclude articles related to robotic surgery or robot rehabilitation from the search results. If available, age (over 65 years old) and language (English and Korean) filters were used. The literature in only English and Korean was searched, and the search period was limited to 10 years, from July 2011 to June 2021, considering the speed of development in robotics. One hundred and fifty-nine documents were assessed for eligibility, and 75 articles were included in the analysis (Fig. 1).

\subsection{Data analysis process}

Articles selected for consideration were evaluated for their validity and quality. A literature review was conducted until the properties of the concepts extracted from 75 articles were saturated. Data collection and analyses were conducted simultaneously. Considering the general flow and the topics covered by the selected literature, nursing literature was analyzed first, followed by medical, computer sciences, engineering, and other academic literature. The analyses were subsequently incorporated to consider the definition of concepts. Consensus and disagreement over concepts between different disciplines were examined, and conceptual changes over time were identified. Subjects repeatedly appearing in the analysis process were categorized into "Attributes," "Antecedents," and "Consequences."

\section{Results}

This section presents and discusses the results in the form of attributes, antecedents, and consequences. Figure 2 depicts the relationship between antecedents, attributes, and consequences. Figure 3 presents the chronological trend of companion robots and similar concepts.

\subsection{Antecedents of companion robots}

Antecedents are events occurring before the occurrence of the intended concept [12]. The antecedents of companion 


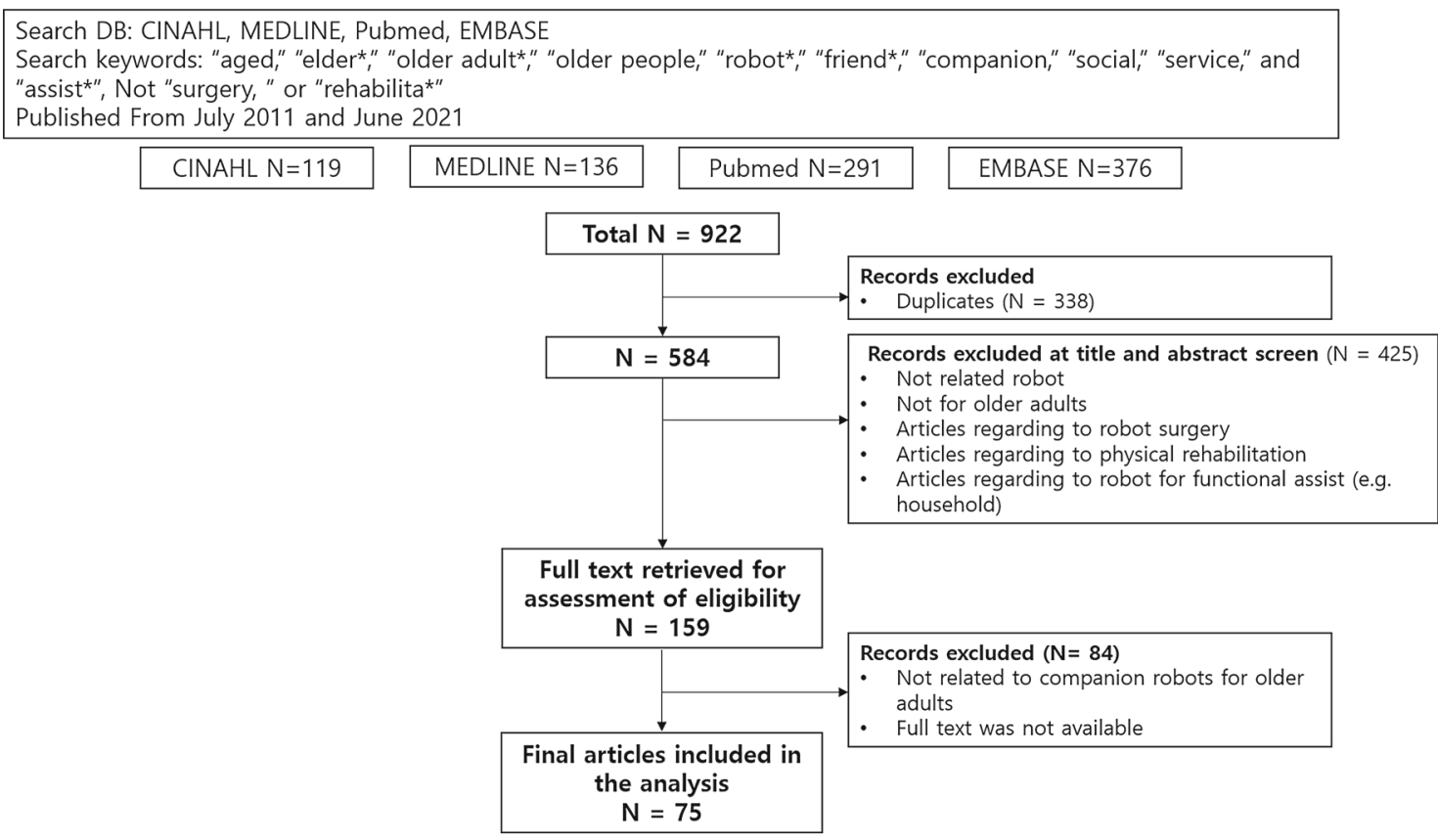

Fig. 1 Flowchart of the article selection process

Fig. 2 Proposed conceptual model of companion robots for older adults

\begin{tabular}{|c|c|c|}
\hline Antecedents & Attributes & Consequences \\
\hline $\begin{array}{l}\text { Individual factors: } \\
\text { cognitive ability, mobility, } \\
\text { physical ability, age and } \\
\text { gender, personal history } \\
\text { or biography, } \\
\text { socioeconomic status } \\
\text { Attitudes toward robots: } \\
\text { the belief that new } \\
\text { technology will make life } \\
\text { better, preparedness to } \\
\text { accept robots, the need } \\
\text { of the application of } \\
\text { robot technology and } \\
\text { willingness }\end{array}$ & $\begin{array}{l}\text { Human-robot interaction } \\
\text { general robotic capabilities such } \\
\text { as autonomous navigation, } \\
\text { manipulation, perception, } \\
\text { assistive specific capabilities, } \\
\text { consciousness and personhood } \\
\text { Function, appearance and } \\
\text { structure of the robot: } \\
\text { coping with emergencies, } \\
\text { physical assistance, stimulation, } \\
\text { assistance in memory recall } \\
\text { including medication, } \\
\text { collaboration with health care } \\
\text { staff, entertainment, equipped } \\
\text { with hardware, software } \\
\text { architecture }\end{array}$ & $\begin{array}{l}\text { User-related consequences : } \\
\text { Improving management, } \\
\text { reducing social isolation and } \\
\text { loneliness, improving social } \\
\text { functioning and quality of } \\
\text { life, independent lifestyle and } \\
\text { aging in place } \\
\text { Caregiver-related } \\
\text { consequences: } \\
\text { Improving quality of life of } \\
\text { caregivers by reducing } \\
\text { caregiver burden, facilitating } \\
\text { social interaction with family } \\
\text { and healthcare providers, } \\
\text { enhancing job satisfaction of } \\
\text { the staff }\end{array}$ \\
\hline $\begin{array}{l}\text { Caregiver and social } \\
\text { factors: } \\
\text { The caregiver's experience } \\
\text { with the use of robots, } \\
\text { support of the } \\
\text { community }\end{array}$ & $\begin{array}{l}\text { Being a companion: } \\
\text { Interact with and sustain a } \\
\text { dialog with the users } \\
\text { Cost and management of the } \\
\text { robot: } \\
\text { A low-cost robot may facilitate } \\
\text { continuous use, periodic } \\
\text { program updates are essential }\end{array}$ & $\begin{array}{l}\text { Health-related } \\
\text { consequences: } \\
\text { The emotional, physical and } \\
\text { cognitive effect } \\
\text { Improving the user's } \\
\text { healthcare outcomes, } \\
\text { reducing social healthcare } \\
\text { cost by lowering the use of } \\
\text { medical resources }\end{array}$ \\
\hline
\end{tabular}

robots relate to individual factors, attitude toward robots, and caregiver and social factors.

\subsubsection{Antecedents related to individual factors}

Individual factors, such as demographics, lifestyles, and personalities, are closely related to companion robots [12].
Health conditions, such as the user's cognitive ability, mobility, physical ability, including comorbidities, were mentioned as important antecedents [2, 6-8, 13-16].

Additionally, older people's personal history or biography can affect how they react and respond to their companion robots. Thus, experience with the robot or pet and the degree of knowledge related to the robot can be antecedents 
Fig. 3 Chronological trend of companion robots and similar concepts

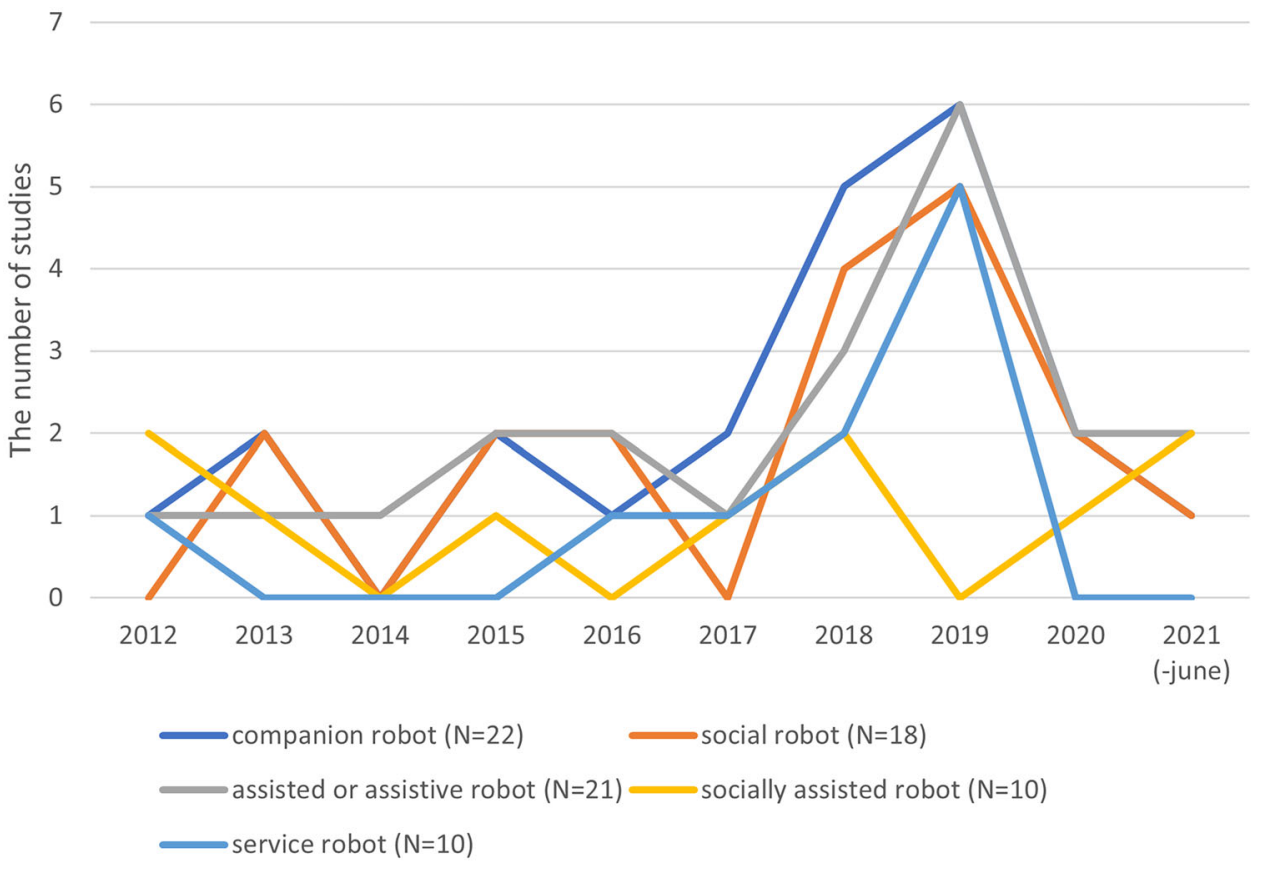

for actual engagement with the robot [6, 7, 17-19]. Furthermore, socioeconomic status, including living environment, former occupation, retirement status, and education level, and informatics competency, including experience using computers or apps and eHealth literacy, were significantly associated with acceptance of robots $[13,15,16$, 20-23].

\subsubsection{Antecedents related to attitude toward robots}

Attitude toward robots, such as the belief that new technologies or devices will make life better, are antecedents of companion robots $[13,14,24,25]$. Considering that older people may find it difficult to adapt to new and advanced technologies, the degree of preparedness to accept robots can affect actual robot acceptance [13]. It has also been emphasized that the need for robotic technology and willingness as an older person may affect acceptance of robots. However, some studies reported that although older adults experience difficulties managing some of their activities, they showed no signs of needing or wanting a robot [26-28]. Many older people are reluctant to change their lifestyle; therefore, new interventions in the form of robots need not change their habits or modify their environment [24, 29, 30].

\subsubsection{Antecedents related to the caregiver and social factor}

Studies reported that the caregiver's experience with the use of robots and support of the community or caregiver in the context of their application were important priori- ties. Caregivers identified numerous opportunities and were more open to robots [28]. However, they have difficulties imagining what robots exactly are because of it being something new and them lacking experience with robotics [31]. Studies found that training from experts skilled in robot use was essential before application [3, 13]. This suggests that even if older adults are trained in using a robot, they need to be explained the process repeatedly to help them adapt to it. Additionally, when using robots for older people in institutions, such as nursing homes, trained staff, other therapies, and a good staff-to-patient ratio should be considered [32].

\subsection{Defining attributes of companion robots}

Specifying the key attributes of a concept is an important part of its clarification, resulting in a deep understanding of the concept and differentiating it from similar or related concepts [12]. The attributes of a companion robot are "human-robot interaction," "function, features, and structure of the robot," "being a companion," and "cost and management of the robot."

\subsubsection{Human-robot interaction (HRI)}

Robots in health care are typically endowed not only with general robotic capabilities, such as autonomous navigation, manipulation, or perception, but also with assistive specific capabilities. Thus, HRI is crucial in this domain of application [33-36]. The authors of [36] mentioned that the use of robots involves concepts of consciousness 
and personhood, and HRI has already become a field of research in which scholars are discovering psychological, philosophical, and even spiritual issues bearing significant implications for traditional nursing values. For example, in a practical application situation, positive behavioral responses were demonstrated through residents' touching, petting, stroking, holding, and hugging the companion robot [6].

To enjoy long-term and engaging multimodal interaction, the human-robot dialog is a key factor, which can be implemented through semantic analysis, understanding emotions from speech analysis, and the design of various robot behaviors $[7,13,37,38]$. These dialogs need to be flexible enough to adapt to unforeseen circumstances during the conversation [38]. The authors of [20] found that older adults want to interact with robots at a moderate level; the robot need not constantly interact with its user but should be available when required. Further, for a successful HRI, the preparation for using a robot should be gradual initially, with considerable involvement of professional personnel to help the user get accustomed to the robot $[13,39]$.

\subsubsection{Function, features, and structure of the robot}

The functions of the robot as presented in previous studies included service-related and companion-related functions, such as coping with emergencies, physical assistance, stimulation, assistance in memory recall, including medication, nutritional support, help locate or bring objects, housekeeping, collaboration with healthcare staff, logging daily activities, and entertainment $[2,6,8,13,19,22,24,33,34$, 37, 40-45]. Early detection and timely reaction to emergencies, including falls, assisting and monitoring older adults using telepresence, and collaborating with healthcare staff, including exchanging data, were mentioned as important functions [19, 24, 33, 37, 46]. Additionally, assistance with mobility or rehabilitation and self-care support through cognitive or emotional stimulation are helpful functions in the life of older adults $[8,13,15,24,33,47]$. They also enjoyed a companion robot with entertainment functions, including dancing, singing, storytelling, playing board games, or news reporting $[15,25]$. According to studies, older adults were more receptive to the use of robots for simple and nonintimate physical assistance, such as reminders, domestic chores, and communication, while they were less receptive to the use of robots in activities, such as taking a bath, toileting, and managing finances [45, 48, 49]. Some studies on assisted robots reported that older adults value servicerelated functions more than companion-related functions [20], and robot functionality is more important than appearance [42].

To perform these functions, a robot needs to be equipped with hardware, such as arms, sensors, cameras, microphones, tablets, monitors, or mobile manipulators [3, 17, 30, 50], and a software architecture capable of navigation, manipulation, and object recognition $[5,17,37,46]$. In other words, robots can listen, respond to speech, talk in a few circumstances, recognize touch, and detect sound and light [5, 19, 38]. Social robots that could learn and adapt to people's preferences, perceptions, and the tasks at hand may fare better than a one-size-fits-all approach [12, 51, 52]. Appearance is also an important trait of companion robots; robots can be human-like, service-oriented, or animal-like. It has been reported that preference for appearance varies widely among older adults: Some prefer animal-like robots, while others want a humanoid robot $[19,20,26,39,45$, 53].

\subsubsection{Being a companion}

The companion robot can interact with and sustain a dialog with the users, thus reducing their loneliness and becoming friends [13, 15, 19, 24, 30, 32, 39, 41, 45, 54]. The terms "Social robot," "Assistive or assisted robot," or "Social assistive robot" were used interchangeably with "Companion robot" in the studies. In a study, a companion robot was defined as an assistant companion with an autonomous intelligence who should understand "user's commands" and how they feel, such as health disposition [54].

Several studies presented advantages of a companion robot, including pet robots, comparing them to pets. There is no need to feed, walk, and clean up the robot; thus, compared to a traditional pet, older adults do not need to take care of companion robots [32], and they are always available without any behavioral irregularities [55]. A companion robot can accompany older adults to the hospital or short-term care, and in case of damage, it can be repaired and replaced with a duplicate [53]. Further, robots yield similar results to complementary therapies with animals, as they generate positive emotions and promote multisensorial interactions through sight, hearing, and touch [56, 57]. The authors of [39] found that residents developed an emotional attachment to the robot in that they expressed affection for the robot and treated it as if they were a real pet. Robots helped combat loneliness by acting as a direct companion, a catalyst for social interaction, facilitating remote communication with others, and reminding users of upcoming social engagements $[25$, 58].

However, many participants worried about the thought of a robot as a companion. Robots that satisfy a companionship role were seen by some as a form of deception, given that such a relationship was thought of as counterfeit [45, 59]. 


\subsubsection{Cost and management of robot}

Until recently, companion robots, including robopets, have been prohibitively expensive [54]. The cost was a common reason for choosing not to purchase a device [60]. A new wave of cheaper robopets or low-cost telepresence robots may facilitate their continuous use $[6,8,17,18,32,43]$. Additionally, periodic program updates and management are essential for long-term interaction [13].

\subsection{Consequences of companion robots}

Consequences are outcomes that occur due to the concept and can shed light on the social context in which the concept is used $[12,61]$. There are user-, caregiver-, and health-related consequences for companion robots.

\subsubsection{User-related consequences}

The findings suggest that the use of robots allows older adults to maintain their independence by improving health management and reducing social isolation and loneliness, thereby significantly contributing to improving their quality of life or well-being [3, 8, 15, 31, 32, 56, 62-67]. Companion robots have been widely used in addressing social isolation-a phenomenon older adults experience more than younger people because of the increased possibility of health problems and life-changing events $[2,62-64,68,69]$. Companion robots can establish a direct relationship with the robot and enhance social contact between the users and the staff and family, thereby improving social functioning, mood, and participation $[6,18,32,50,66]$.

Current healthcare and social policies encourage the concept of "aging in place," where older people remain in their own residences and communities, as this is the best solution in terms of health, quality of life, and social connections of older people as well as in economic terms [8]. Companion robots may have value in supporting older adults in having an independent lifestyle and autonomy in terms of "aging in place" [19]. In other words, companion robots may enhance the ambient assisted living capabilities [33], thus prolonging independent living $[17,70]$. For long-term usage, identifying features that enhance older adults' acceptance of robots is essential $[20,25,33,60,63]$.

\subsubsection{Caregiver-related consequences}

Companion robots may improve caregivers' quality of life by reducing their burdens. Specifically, the companion robot can contribute to reducing time, level of assistance, and the energy invested into caregiving, thus decreasing anxiety, fear, and task difficulty as well as increasing the safety of performing activities that require physical assistance $[6,8,40]$.
The telepresence function of robots can assist healthcare providers in everyday activities [18], make access to medical care convenient for healthcare providers and patients [40], and facilitate social interaction with family and healthcare providers [37]. Moreover, companion robots can enhance the staff's job satisfaction [18]. Abbott et al. (2019) found that the staff and family considered companion robots a therapeutic toolbox, convenient, and wonderful support [6]. In contrast, a family's psychological burdens and time restrictions should be considered if family caregivers act as remote robot operators [71].

\subsubsection{Health-related consequences}

Based on literature analysis, the emotional, physical, and cognitive effects of companion robots can be expected to ultimately improve the user's healthcare outcomes and reduce social healthcare costs by lowering medical resource usage $[5,6,18,19,32,34,43,70,72,73]$. Companion robots may be a useful tool in mitigating depression, agitation, anxiety, and loneliness while enhancing social connectedness, engagement, interaction, resilience, and overall quality of life for people with dementia $[65,72,73]$. For instance, companion robots significantly improve facial expressions (affect) and communication with staff (social interaction) [73]. One study reported on the esthetic appeal of the companion robot and how they engaged the residents' visual, tactile, and auditory senses [6].

Studies have reported that behavioral and psychological symptoms of dementia can be reduced through cognitive interaction with the robot $[18,19,34,43]$. As for the physical effects, Bates (2019) reported that the robot could increase the immune system response and reduce psychoactive and pain medications [32]. Furthermore, companion robots can improve healthcare outcomes and have economic effects. Economic effects, such as reduced hospitalization, healthcare costs, and individual and societal costs of caring, have already been reported $[8,40,74,75]$.

\subsection{Barriers and ethical issues in the adoption of companion robots}

Studies reported several concerns regarding robots in general. For instance, the complexity of using the robots and technical obstacles impede implementation in the real world $[49,69]$. Older adults might fear the inability to handle the malfunctioning and rusting of the robot [20]. Further, robots may increase agitation or result in less social contact and more isolation [8, 18, 23, 31, 32]. Residents could also display excessive attachment to the robopets with detrimental effects for the individual and their relationships with other residents. Previous studies reported that residents refused to interact with the robot because of the failure to match 
their expectations or desires [6, 19, 23]. In some cases, people soon lose interest in the non-living robot and its limited behavioral repertoire [53]. The benefits of robots for society and empowerment of older people must be contrasted against possible ethical concerns in the field around issues, such as deceit, infantilization, increased feeling of objectification or anxiety, loss of control and dignity, loss of privacy and personal freedom as well as reduced human contact and accountability [3, 14, 45, 49, 59, 60, 69, 75]. Concerns included the loss of jobs and personal care, while perceived benefits included allowing staff to spend quality time with residents and helping residents with self-care $[3,31,41,42]$.

\section{Discussion}

This study aimed at providing a clear definition of the concept of companion robots by specifying its diverse aspects. Rodgers' (1989) evolutionary concept analysis was used to conduct a literature review and analysis, describing the companion robot, to identify relevant precedents, attributes, and consequences based on users, place, purposes, and context [76]. The results showed that this concept is not limited to social robots used for entertainment or assistive robots used for simple tasks and that its proper implementation requires the consideration of many factors, including the needs of users and their family caregivers, the physical and functional abilities of older adults, the purpose of using robots, and the environment where the robot will be used. Although studies have shown that companion robots have been used for various purposes in different times and contexts, every study has emphasized specific aspects and outcomes. Furthermore, the literature review showed that although the development and use of companion robots are practically associated with many challenges, they are valuable, as shown by the abovementioned consequences.

The findings indicated that companion robots are designed to enhance psychological and physical well-being, quality of life, and independence by providing companionship and assisting daily life. This mainly includes interaction, cognitive and social support, mobility support, stimulation, relaxation, reduce stress, health monitoring, and self-care support. Companion robots in the shape of a pet or a humanoid can listen, recognize speech and touch, and detect sound for smooth HRI; telepresence robots aid family caregivers as well as healthcare providers.

HRI was the first attribute considered in companion robots, with studies emphasizing that HRI is critical for long-term use $[6,7,13,46]$. A study reported that there are differences in the preferences regarding HRI between older adults and roboticists [60]. Older adults responded positively toward life-simulation features, eye contact, robot personalization, and obeying commands, and these are features undervalued by roboticists [60]. It proposed paying greater attention to users' preferences for robots' designs and features. Cultural, physical and social environment and application of longitudinal and simultaneous examination of uses, outcomes, and constraints should be considered in the robot design and development phase [35].

Despite the importance and high priority of interaction between users and companion robots [46], studies revealed challenges concerning facial or voice recognition, technical or usability issues (e.g., navigation failure, noise level, power consumption, and disconnecting), and the practicalities and modularity in real-life settings [3, 5, 23, 25, 31, 63, 64, 69]. Some studies showed that the robot was considered more like a toy than a supportive device for independent living because of a lack of technological robustness and slow performance [72]. Improvement of social skills and practicalities, including resolution of technical issues, were suggested to promote long-term use.

The second attribute considered the function, features, and structure of the robot. This is also closely related to the interaction between the users and the robot. Robots fulfill several functional and structural needs for healthcare support functions, such as timely reaction to emergencies and assisting and monitoring older adults through the telepresence of healthcare providers $[3,24,50,62]$. The need for robots to perform functions closely related to health care is considered because older adults prefer aging in place to living in an institution or hospital.

Technology is not a device that helps to carry out specific tasks. However, it seems that technical ambitions still guide robots' development and not older adults' requirements [52]. Needs and capabilities vary across users [26, 29]. To be truly effective, the technologies need to be integrated into existing health and social contexts with the final aim to fit into a personalized and comprehensive older adults' everyday life $[8,19,23,24,26,45,51,68]$. This emphasizes the complexity of service robot development for older adults and highlights the need for a personalized and flexible solution [25, 51]. Familiar product forms with augmented product functionalities would fit the system and maximize early product adoption [26].

Being a companion was the third attribute. "Being a friend and helper" was the most desired trait of a robot service [19], and the robot can live with the older adults and become a conversation partner, thus forming a friendship [18]. This can help combat loneliness, a major social issue, thus making it a good support system for aging in place.

However, there is another viewpoint according to which informal care will become increasingly important in future and will not be replaced by companion robots [31, 36, 77]. Caring has a subjective and sentient dimension and is not confined to performing tasks. A study that examined the 
opinion of future healthcare professionals regarding the use of assistive robots reported that the robot should not be an older person's companion but only act as an assistant [41]. Older adults were not straightforwardly rejecting robotic assistance; they believed that robots could supplement human assistance in some ways [77]. This implies that robots should not aim to replace humans but should perform certain tasks. Robots could be introduced in the environment of nursing practice as assistants, supplements, and complements [36]. In this situation, healthcare providers should call for more specific definitions of their intended role of the robot. In a study, nurses responded negatively about using robots for nursing care; however, they were generally positive about their use for service, monitoring, and communication tasks [78]. For healthcare providers, including nurses, openness is required to not only accept but also embrace and contribute to the design of the technology brought to market [79].

Despite older adults encountering difficulties in many activities of daily life, a robot has been judged as an interesting solution only in some circumstances [20,71]. Older adults worry about being bothered by companion robots while resting; however, experts think that older adults might feel lonely and need companionship in these situations [80]. A similar study showed that older adults are more positive toward the listening robot than the speaking robot [81]. Monitoring and managing emergencies, helping with reaching, fetching, and carrying objects that are too heavy or positioned in unreachable places: These are tasks for which robotic support has been widely accepted, while tasks involving direct physical contact between the person and the robot are not appreciated [71]. This is also related to the ethical issue where it is necessary to provide older people the authority to choose tasks to be entrusted to the robot.

Currently, limited evidence demonstrates that robots enable aging people to continue living at home [82]. Recent reviews have reported that many technological intervention studies targeting older people lack quantitative outcomes as a part of their evaluation process, and there is a lack of consensus as to which outcomes to use [32, 83]. Several studies were conducted in laboratories and hospital clinics. For robots supporting independent living of older adults, most were found to be under development or in the concept phase, and only a few robots turned to be commercially available [51]. Further, existing research focuses on the internal validity of robots. There is significantly less research investigating their external validity, such as organizational or wider contextual factors affecting their implementation in real-world practice [69]. More research is required to confirm the usefulness of real-world applications in older adults' residences.

Psychosocial and ethical issues, cost, safety issues, and intervention time may impede the broad use of technology in older age. Some ethical guidelines and practical comments are available for roboticists $[45,59,75]$. The summoning of an internal ethics board can help facilitate this process, especially where users' dignity in connection with tasks the robot fulfills or interaction with a robot are concerned [59]. Involving staff and/or relatives early on can help identify ethical issues, misunderstandings, and requirements and expectations. Older adults and their autonomy must be respected by giving them choices regarding robots and their functions [45]. If the interventions change their lifestyle significantly, it is imperative to help them adapt to robots gradually. To avoid deception, informing older adults in a comprehensible way about the real capabilities and limitations of robots is essential $[45,59]$. Informed consent is one tool to address privacy issues [75], and no-go zones should be defined with users and caregivers before the actual deployment [59].

It is also necessary to prepare for high purchasing costs, updates, and failure for the protection of services as well as their long-term availability. Roboticists and manufacturers can use a risk assessment assistant tool for companion robots based on hazard scenarios [75, 84]. An integrated, multidisciplinary approach could be the solution. Successful technology development requires great effort in interdisciplinary collaboration. The involved teams include clinicians, social and behavioral scientists, physiotherapists and psychotherapists, engineers, computer scientists, designers, end-users, and policy experts $[8,52]$.

The limitation of the present study lies in the fact that the researchers only reviewed studies published in English and Korean and full texts of which were available.

\section{Conclusion}

This study systematically analyzed the definition and described the concept of a companion robot for older adults. The objective of this analysis was to provide conceptual clarity and direction for future research and advance knowledge for formal and informal caregivers by providing an operational definition of the companion robot. A better understanding of the concept will assist caregivers in implementing interventions where necessary.

By defining attributes, antecedents, and consequences of the companion robot through a concept analysis, conceptual clarity has been obtained. It is necessary to consider users' needs, functional ability, and usage context of the robot. A great effort in interdisciplinary collaboration is necessary to integrate technology into existing health and social service systems. The conceptual clarity provided by this analysis will inform future decision making and interventions by caregivers for aging in place. The concept of companion robots for older adults can underpin future research into companion robots for different purposes and in different contexts. 
Funding This work was supported by Seoul National University Research Grant in 2019. This work was supported by the Gachon University Research Fund of 2020 (GCU-2020-02130001).

\section{References}

1. Harrington L, Heidkamp M (2013) The aging workforce: Challenges for the health care industry workforce. Aging 2020:115

2. Shishehgar M, Kerr D, Blake J (2019) The effectiveness of various robotic technologies in assisting older adults. J Health Inform 25:892-918. https://doi.org/10.1177/1460458217729729

3. Isabet B, Pino M, Lewis M et al (2021) Social telepresence robots: A narrative review of experiments involving older adults before and during the COVID-19 pandemic. Int J Environ Res Pub He 18(7):3597

4. Truxillo DM, Cadiz DM, Hammer LB (2015) Supporting the aging workforce: a review and recommendations for workplace intervention research. Annu Rev Organ Psychol Organ Behav 2:251-381. https://doi.org/10.1146/annurev-orgpsych-032414-111435

5. Zsiga K, Tóth A, Pilissy T et al (2018) Evaluation of a companion robot based on field tests with single older adults in their homes. Assist Technol 30(5):259-266

6. Abbott R, Orr N, McGill P et al (2019) How do "robopets" impact the health and well-being of residents in care homes? A systematic review of qualitative and quantitative evidence. Int J Older People Nursing 14:12239. https://doi.org/10.1111/opn.12239

7. Bemelmans R, Gelderblom GJ, Jonker P, de Witte L (2012) Socially assistive robots in elderly care: a systematic review into effects and effectiveness. J Am Med Dir Assoc 13:114-120. https://doi.org/ 10.1016/j.jamda.2010.10.002

8. Pilotto A, Boi R, Petermans J (2018) Technology in geriatrics. Age Ageing 47:771-774. https://doi.org/10.1093/ageing/afy026

9. Rodgers BL, Knafl KA (2000) Concept development in nursing: foundations, techniques, and applications. Saunders, Philadelphia

10. Wilson J (1969) Thinking with concepts. Cambridge University Press, Cambridge. (Original work published 1963)

11. Tofthagen R, Fagerstrøm LM (2010) Rodgers' evolutionary concept analysis - a valid method for developing knowledge in nursing science. Scand J Caring Sci 24:21-31

12. Walker LO, Avant KC (2010) Concept analysis. In: Walker LO, Avant KC (eds) Strategies for theory construction in nursing. Pearson, London

13. Tobis S, Salatino C, Tapus A, Suwalska A (2017) Robots in care for older people: opinions of potential end-users. Eur Geriatr Med 8:S31

14. Zsiga K, Edelmayer G, Rumeau P et al (2013) Home care robot for socially supporting the elderly: focus group studies in three European countries to screen user attitudes and requirements. Int J Rehabil Res 36:375-378. https://doi.org/10.1097/ MRR.0b013e3283643d26

15. Chiu CJ, Hsieh S, Li CW et al (2021) Needs and preferences of middle-aged and older adults in Taiwan for companion robots and pets: survey study. J Med Internet Res 23(6):e23471

16. Granata C, Pino M, Legouverneur G et al (2013) Robot services for elderly with cognitive impairment: testing usability of graphical user interfaces. Technol Health Care 21(3):217-231

17. Koceska N, Koceski S, Beomonte ZP et al (2019) A telemedicine robot system for assisted and independent living. Sensors 19:834. https://doi.org/10.3390/s19040834

18. Moyle W, Bramble M, Jones C, Murfield J (2018) Care staff perceptions of a social robot called Paro and a look-alike plush toy: a descriptive qualitative approach. Aging Ment Health 22:330-335. https://doi.org/10.1080/13607863.2016.1262820
19. Shishehgar M, Kerr D, Blake J (2018) A systematic review of research into how robotic technology can help older people. Smart Health 7-8:1-18. https://doi.org/10.1016/j.smhl.2018.03.002

20. Chu L, Chen HW, Cheng PY et al (2019) Identifying features that enhance older adults' acceptance of robots: a mixed methods study. Gerontology 65:441-450. https://doi.org/10.1159/000494881

21. Sung HC, Chang SM, Chin MY, Lee WL (2015) Robot-assisted therapy for improving social interactions and activity participation among institutionalized older adults: a pilot study. Asia Pac Psychiatry 7:1-6. https://doi.org/10.1111/appy.12131

22. Daniele K, Marcucci M, Cattaneo C et al (2019) How prefrail older people living alone perceive information and communications technology and what they would ask a robot for: qualitative study. J Med Internet Res 21(8):13228

23. Pino M, Boulay M, Jouen Fet al (2015) Are we ready for robots that care for us? Attitudes and opinions of older adults toward socially assistive robots. Front Aging Neurosci 7:141

24. Park YH, Chang HK, Lee MH, Lee SH (2019) Communitydwelling older adults' needs and acceptance regarding the use of robot technology to assist with daily living performance. BMC Geriatr 19:1-9. https://doi.org/10.1186/s12877-019-1227-7

25. Gasteiger $\mathrm{N}$ et al (2021) Older adults' experiences and perceptions of living with Bomy, an assistive dailycare robot: a qualitative study. Assist Technol. https://doi.org/10.1080/10400435.2021.1877210

26. Wu YH, Fassert C, Rigaud AS et al (2012) Designing robots for the elderly: appearance issue and beyond. Arch Gerontol Geriatr 54(1):121-126

27. Wu YH, Cristancho-Lacroix V, Fassert C et al (2016) The attitudes and perceptions of older adults with mild cognitive impairment toward an assistive robot. J Appl Gerontol 35(1):3-17

28. Wang RH, Sudhama A, Begum M et al (2017) Robots to assist daily activities: Views of older adults with Alzheimer's disease and their caregivers. Int Psychogeriatr 29(1):67-79

29. Doering N, Richter K, Gross HM et al (2015) Robotic companions for older people: a case study in the wild. Stud Health Technol Inform 219:147-152

30. Eftring H, Frennert S (2016) Designing a social and assistive robot for seniors. Z Gerontol Geriatr 49(4):274-281

31. D'Onofrio G, Fiorini L, Hoshino H et al (2019) Assistive robots for socialization in elderly people: results pertaining to the needs of the users. Aging Clin Exp Res 31(9):1313-1329

32. Bates M (2019) Robotic pets: a senior's best friend? IEEE Pulse 10:17-20. https://doi.org/10.1109/MPULS.2019.2922565

33. Gomez-Donoso F, Escalona F, Rivas FM et al (2019) Enhancing the ambient assisted living capabilities with a mobile robot. Comput Intell Neurosci. https://doi.org/10.1155/2019/9412384

34. Saez-Pons J, Syrdal DS, Dautenhahn K (2015) What has happened today? Memory visualisation of a robot companion to assist user's memory. J Assist Technol 9:207-218. https://doi.org/10.1108/JAT02-2015-0004

35. Zafrani O, Nimrod G (2019) Towards a holistic approach to studying human-robot interaction in later life. Gerontologist 59(1):e26-e36

36. Metzler TA, Barnes SJ (2014) Three dialogues concerning robots in elder care. Nurs Philos 15:4-13. https://doi.org/10.1111/nup. 12027

37. Lebec O, Ben Ghezala MW, Leynart V et al (2013) High level functions for the intuitive use of an assistive robot. IEEE Int Conf Rehabil Robot. https://doi.org/10.1109/ICORR.2013.6650374

38. Fernández-Rodicio E, Castro-González Á, Alonso-Martín F et al (2020) Modelling multimodal dialogues for social robots using communicative acts. Sensors 20(12). https://doi.org/10.3390/ s20123440

39. Robinson H, MacDonald B, Kerse N, Broadbent E (2013) The psychosocial effects of a companion robot: a randomized controlled 
trial. J Am Med Dir Assoc 14:661-667. https://doi.org/10.1016/j. jamda.2013.02.007

40. Mapundu Z, Simonnet T, van der Walt JS (2012) A videoconferencing tool acting as a home-based healthcare monitoring robot for elderly patients. Stud Health Technol Inform 182:180-188

41. Chen SC, Jones C, Moyle W (2018) Social robots for depression in older adults: A systematic review. J Nurs Scholarsh 50:612-622. https://doi.org/10.1111/jnu.12423

42. Broadbent E, Tamagawa R, Patience A et al (2012) Attitudes towards health-care robots in a retirement village. Australas J Ageing 31(2): $115-120$

43. Sefcik JS, Johnson MJ, Yim M et al (2018) Stakeholders' perceptions sought to inform the development of a low-cost mobile robot for older adults: a qualitative descriptive study. Clin Nurs Res 27(1):61-80

44. Lukasik S, Tobis S, Wieczorowska-Tobis K et al (2018) Could robots help older people with age-related nutritional problems? Opinions of potential users. Int J Environ Res Pub He 15(11):2535

45. Vandemeulebroucke T, de Casterlé BD, Gastmans C et al (2018) How do older adults experience and perceive socially assistive robots in aged care: a systematic review of qualitative evidence. Aging Ment Health 22(2):149-167

46. Korchut A, Szklener S, Abdelnour C et al (2017) Challenges for service robots-requirements of elderly adults with cognitive impairments. Front Neurol 8:228

47. Góngora Alonso S, Hamrioui S, de la Torre DI et al (2019) Social robots for people with aging and dementia: a systematic review of literature. Telemed J E Health 25(7):533-540

48. Huang T, Huang C (2021) Attitudes of the elderly living independently towards the use of robots to assist with activities of daily living. Work 69(1):55-65

49. Robillard JM, Kabacińska K (2020) Realizing the potential of robotics for aged care through co-creation. J Alzheimer's Dis 76(2):461-466

50. Moyle W, Arnautovska U, Ownsworth T et al (2017) Potential of telepresence robots to enhance social connectedness in older adults with dementia: an integrative review of feasibility. Int Psychogeriatr 29(12):1951-1964

51. Bedaf S, Marti P, De Witte L et al (2019) What are the preferred characteristics of a service robot for the elderly? A multi-country focus group study with older adults and caregivers. Assist Technol 31(3):147-157

52. Bedaf S, de Witte L (2017) Robots for elderly care: their level of social interactions and the targeted end user. Stud Health Technol Inform 242:472-478

53. Preuß D, Legal F (2017) Living with the animals: animal or robotic companions for the elderly in smart homes? J Med Ethics 43(6):407-410

54. Ozdemir D, Cibulka J, Stepankova O et al (2021) Design and implementation framework of social assistive robotics for people with dementia-a scoping review. Health Technol 11(2):367-378

55. Valenti Soler M, Agüera-Ortiz L, Olazarán Rodríguez J et al (2015) (2015) Social robots in advanced dementia. Front Aging Neurosci 7:133

56. Barata AN (2019) Social robots as a complementary therapy in chronic, progressive diseases. In: Sequeira JS (eds) Robotics in healthcare: field examples and challenges. Springer, Berlin, pp 95-102

57. Hudson J, Ungar R, Albright L et al (2020) Robotic pet use among community-dwelling older adults. J Gerontol B Psychol Sci Soc Sci 75(9):2018-2028

58. Marti P, Stienstra JT (2013) Exploring empathy in interaction. GeroPsych 26(2):101-112

59. Körtner T (2016) Ethical challenges in the use of social service robots for elderly people. Z Gerontol Geriatr 49(4):303-307
60. Bradwell HL, Winnington R, Thill S et al (2020) Ethical perceptions towards real-world use of companion robots with older people and people with dementia: survey opinions among younger adults. BMC Geriatr 20(1):244

61. Cronin P, Ryan F, Coughlan M (2010) Concept analysis in health care research. Int J Ther Rehabil 17:62-68

62. Koceski S, Koceska N (2016) Evaluation of an assistive telepresence robot for elderly healthcare. J Med Syst 40(5):121

63. Casey D, Barrett E, Kovacic T. et al (2020) The perceptions of people with dementia and key stakeholders regarding the use and impact of the social robot MARIO. Int J Environ Res Public Health 17(22):8621

64. Inoue K, Sasaki C, Nakamura M (2015) Communication robots for elderly people and their families to support their daily lives-case study of two families living with the communication robot. Stud Health Technol Inform 217:980-983

65. D'Onofrio G, Sancarlo D, Raciti M et al (2019) MARIO project: Validation and evidence of service robots for older people with dementia. J Alzheimer's Dis 68(4):1587-1601

66. Gustafsson C, Svanberg C, Müllersdorf M et al (2015) Using a robotic cat in dementia care: A pilot study. J Gerontol Nurs 41(10):46-56

67. Darragh M, Ahn HS, MacDonald B et al (2017) Homecare robots to improve health and well-being in mild cognitive impairment and early stage dementia: results from a scoping study. J Am Med Dir Assoc 18(12):1099.e1091-e1099

68. Khosla R, Chu MT, Khaksar SMS et al (2021) Engagement and experience of older people with socially assistive robots in home care. Assist Technol 33(2):57-71

69. Koh WQ, Felding SA, Toomey E et al (2021) Barriers and facilitators to the implementation of social robots for older adults and people with dementia: a scoping review. BMC Geriatr 21(1):351

70. Van Patten R, Keller AV, Maye JE et al (2020) Home-based cognitively assistive robots: maximizing cognitive functioning and maintaining independence in older adults without dementia. Clin Interv Aging 15:1129-1139

71. Pigini L, Facal D, Blasi L et al (2012) Service robots in elderly care at home: Users' needs and perceptions as a basis for concept development. Technol Disabil 24(4):303-311

72. Pripfl J, Körtner T, Batko-Klein D et al (2016) Social service robots to support independent living: experiences from a field trial. Z Gerontol Geriatr 49(4):282-287

73. Liang A, Piroth I, Robinson H et al (2017) A pilot randomized trial of a companion robot for people with dementia living in the community. J Am Med Dir Assoc 18:871-878. https://doi.org/10. 1016/j.jamda.2017.05.019

74. Portacolone E, Halpern J, Luxenberg J et al (2020) Ethical issues raised by the introduction of artificial companions to older adults with cognitive impairment: A call for interdisciplinary collaborations. J Alzheimer's Dis 76(2):445-455

75. Lukasik S, Tobis S, Kropińska S et al (2020) Role of assistive robots in the care of older people: survey study among medical and nursing students. J Med Internet Res 22(8):e18003

76. Rodgers BL (1989) Concepts, analysis and the development of nursing knowledge: the evolutionary cycle. J Adv Nurs 14:330-335

77. Lehoux P, Grimard D (2018) When robots care: public deliberations on how technology and humans may support independent living for older adults. Soc Sci Med 211:330-337

78. Goransson O, Pettersson K, Larsson PA (2008) Lennernas B (2008) Personnel's attitudes toward robot assisted health care-a pilot study in 111 respondents. Stud Health Technol Inform 137:56-60

79. Sharts-Hopko NC (2014) The coming revolution in personal care robotics: What does it mean for nurses? Nurs Adm Q 38(1):5-12

80. Chen N, Song J, Li B (2019) Providing aging adults social robots' companionship in home-based elder care. J Healthc Eng 2019:2726837 
81. Hirano M, Ogura K, Sakamoto D et al (2019) Robotic utterance style to promote conversation with older people in Japan. Gerontechnology 18(2):89-96

82. Pearce AJ, Adair B, Miller K et al (2012) Robotics to enable older adults to remain living at home. J Aging Res 2012:538169

83. Mois G, Beer JM (2020) The role of healthcare robotics in providing support to older adults: a socio-ecological perspective. Curr Geriatr Rep 9(2):82-89
84. Keiko H, Osamu M (2017) Development of a risk assessment assistance tool for robotic care devices. Stud Health Technol Inform 242:551-557

Publisher's Note Springer Nature remains neutral with regard to jurisdictional claims in published maps and institutional affiliations.

\section{Author and Affiliations}

\section{Jeongeun $\mathrm{Kim}^{1}$. Sukwha Kim² ${ }^{2}$ Seongheui $\mathrm{Kim}^{3}$ - Euehun Lee ${ }^{4}$ Yoonjeong $\mathrm{Heo}^{5}$. Cheol-Yong Hwang ${ }^{6}$. Yun-Young Choi ${ }^{7} \cdot$ Hyoun-Joong Kong ${ }^{8} \cdot$ Hyeongju Ryu ${ }^{9} \cdot$ Hyeongsuk Lee ${ }^{10}$}

Jeongeun Kim

kim0424@snu.ac.kr

Sukwha Kim

kimsw@snu.ac.kr

Seongheui Kim

hemyeong@snu.ac.kr

Euehun Lee

danbee91@kaist.ac.kr

Yoonjeong Heo

hyjkmg@snu.ac.kr

Cheol-Yong Hwang

cyhwang@snu.ac.kr

Yun-Young Choi

melusine@snu.ac.kr

Hyoun-Joong Kong

gongcop@melab.snu.ac.kr

Hyeongju Ryu

rnyoo@snu.ac.kr

1 Research Institute of Nursing Science, Seoul National University, Seoul, Republic of Korea
2 Department of Reconstructive Plastic Surgery, Medical Big Data Research Center, Seoul National University College of Medicine, Seoul, Republic of Korea

3 College of Fine Arts, Seoul National University, Seoul, Republic of Korea

4 Korea Advanced Institute of Science and Technology, Daejeon, Republic of Korea

5 College of Music, Seoul National University, Seoul, Republic of Korea

6 College of Veterinary Medicine, Seoul National University, Seoul, Republic of Korea

7 College of Humanities, Seoul National University, Seoul, Republic of Korea

8 Transdisciplinary Department of Medicine and Advanced Technology (TDMAT), Seoul National University Hospital, Seoul, Republic of Korea

9 Seoul National University Hospital, Seoul, Republic of Korea

10 College of Nursing, Gachon University, Seoul, Republic of Korea 